\title{
Piezoelectric-fluid interaction finite element analysis of a valve-less piezoelectric pump
}

\author{
Y. Uetsuji ${ }^{1}$, H. Kuramae ${ }^{2}$ \& K. Tsuchiya ${ }^{3}$ \\ ${ }^{1}$ Department of Mechanical Engineering, \\ Osaka Institute of Technology, Japan \\ ${ }^{2}$ Department of Technology Management, \\ Osaka Institute of Technology, Japan \\ ${ }^{3}$ Department of Precision Engineering, Tokai University, Japan
}

\begin{abstract}
In this paper, a new composite actuator, which consists of a bimorph piezoelectric plate and a metal cap, was proposed for micro-TAS (Total Analysis Systems) which is widely used for chemical, biological and medical devises. The main objective of this work is to verify the displacement amplification introduced by a new design of composite actuator. At first, the proposed discshaped composite actuator was modeled and the static deflection was compared with conventional ones which are monomorph, bimorph actuators and a composite actuator called "Moonie". Additionally, the proposed composite actuator was applied to a valve-less pump in a Y-shaped fluid channel and a piezoelectric-fluid interaction finite element simulation was performed. Computations demonstrated the superior performance of the new composite actuator compared with conventional ones.
\end{abstract}

Keywords: piezoelectric actuator, valve-less pump, piezoelectric-fluid interaction, finite element analysis.

\section{Introduction}

In recent years, piezoelectric and electrostrictive materials have been used in many actuator applications. Particularly a lot of piezo-actuated MEMS (Micro Electro Mechanical Systems) have been developed by micro and nanotechnology. They are widely used for automobile, information technology 
devises and their application is expanding to chemical, biological and medical devises called micro-TAS.

Piezoelectric actuators can be classified into three types. One is monomorph actuator consisting of a piezoelectric ceramic plate. If the electric filed is applied in the thickness direction, the monomorph actuator extends along the thickness direction and shrivels along in-plane directions. Next is unimorph actuator consisting of a piezoelectric ceramic plate and a metal thin plate. The unimorph actuator is deflected by the difference of in-plane strain between the piezoelectric ceramic plate and the metal thin plate. The other is bimorph actuator composed of two thin plates of piezoelectric ceramic elements bonded together. The bimorph actuator reverses the deformation of two piezoelectric plates by opposite electric filed and it leads to generation of a large deflection.

In order to amplify the displacement of piezoelectric actuators, a composite actuator called "Moonie" was design and fabricated [1-3]. It was a monomorph piezoelectric actuator bonded with metal cap. The Moonie actuator made the metal cap deform by utilizing the in-plane displacement generated by the monomorph piezoelectric actuator. The Moonie actuators are followed by many advanced designs and improvements of geometry and configuration of metal cap $[4,5]$. Hitherto only monomorph structure has been employed for Moonie actuator although bimorph structure is advantageous to a generation of large inplane displacement. It is a lack to investigate the superiority of bimorph actuator for Moonie actuator.

On the other hand, one of key devices in micro-TAS is an electroactive pump to send sample fluid. A valve-less pump becomes one of recent trends and has been developed with various actuating principles, such as electroelasticity, shape memory alloy and electromagneticity as well as piezoelectricity. The piezoelectric actuation has advantages of low power consumption, good reliability and energy efficiency. Piezoelectric pump can be classified into two types. One is unimorph actuators [6, 7] and the other is bimorph actuators $[8,9]$. As the pumping performance is improved according to increasing volumetric change caused by piezoelectric actuation, large-deflection actuators are advantageous to pump. However the Moonie actuator, which can generate lager deflection than unimorph and bimorph actuators, has not been applied to pump.

Consequently, the objective of this work is the proposition of a new composite actuator and its application to the valve-less pump. First, a bimorph actuator is applied to Moonie actuator on behalf of a monomorph actuator. Namely a composite actuator consisting of the bimorph actuator and metal cap is proposed to amplify the deflection. Second, Moonie actuator and the proposed composite actuator are applied to the valve-less pump in Y-shaped fluid channel [9]. The static and dynamic performances of piezoelectric actuators and pump are verified through piezoelectric-fluid interaction finite element simulation. 


\section{Design of composite actuator}

\subsection{Working principle}

Figure 1 shows configuration and deformation features of piezoelectric actuators. In this paper we focus on two conventional actuators and two composite actuators. The former actuators are (a) monomorph and (b) bimorph actuators. In

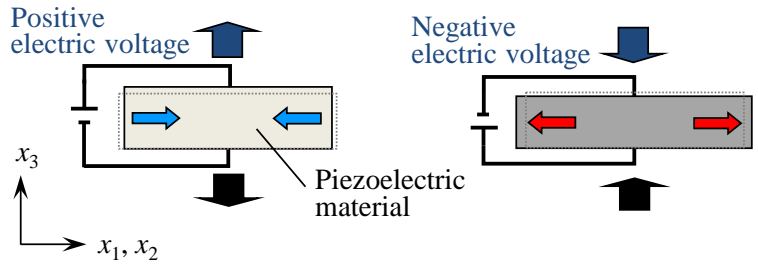

(a) Monomorph actuator

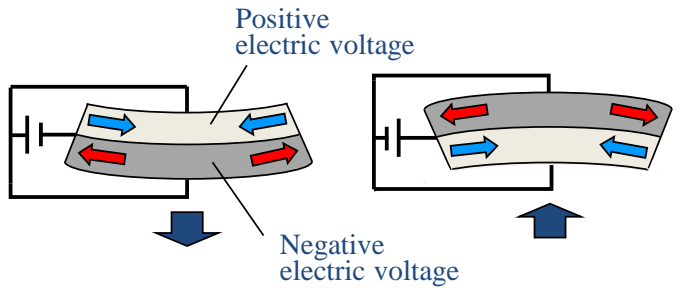

(b) Bimorph actuator

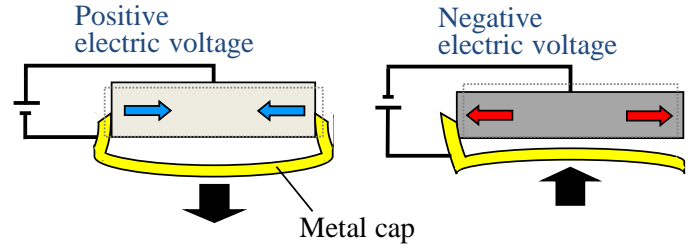

(c) Moonie actuator

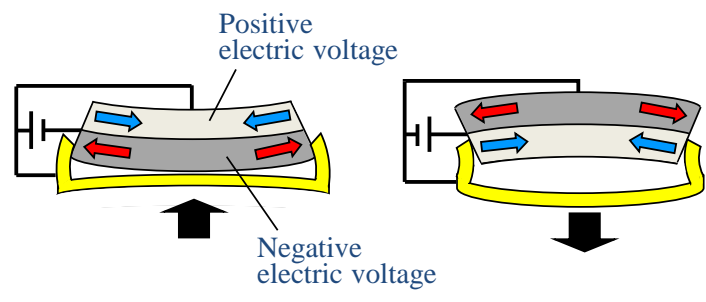

(d) New Moonie actuator

Figure 1: $\quad$ Schematic of deformation features of piezoelectric actuators. 
the case that a positive electric field $\left(E_{3}\right)$ is applied in the thickness direction to the monomorph actuator, it extends in the thickness direction and shrivels in inplane directions. If employing piezoelectric strain constant $d_{\text {mij }}$, mechanical strains, $\varepsilon_{33}$ and $\varepsilon_{11}\left(=\varepsilon_{22}\right)$ can be expressed as $d_{333} E_{3}$ and $d_{311} E_{3}$, respectively. In the case of a negative positive electric field, the deformation is reversed. To get volume change in piezoelectric pump, the out-of-plane strain $\varepsilon_{33}$ caused by piezoelectric strain constant $d_{333}$ is utilized. On the other hand, in the case of the bimorph actuator, the difference of in-plane strains $\varepsilon_{11}$ and $\varepsilon_{22}$ caused by $d_{311}$ and $d_{322}$ between the upper and the lower piezoelectric plates generates large deflection. Out-of-plane and in-plane displacements of the bimorph actuator become larger compared with the monomorph actuator. Additionally, deflection angle occurs at the edge of the bimorph actuator.

The latter actuators are (c) Moonie and (d) the proposed actuators. Both are composite actuators consisting of a piezoelectric element and a metal cap. In the case of the conventional Moonie actuator, the metal cap is bended by in-plane strains caused by the monomorph actuator. The deflection of the metal cap can be amplified if in-plane strains of the piezoelectric element increases. In the case of the proposed composite actuator, the bimorph actuator, whose in-plane strains are larger than monomorph one, is applied to the piezoelectric element of Moonie actuator. Below we call the proposed composite actuator "new Moonie". The new Moonie actuator can amplify the overall deflection by not only larger in-plane strains but also deflection angle at the edge. It is a characteristic of the new Moonie actuator that the plus and minus of deflection are differ between the piezoelectric element and the metal cap. It is important to set a clearance of the metal cap enough large not to intervene in the piezoelectric element.

\subsection{Modeling and analysis of new composite actuator}

To check the deformation behaviour of the proposed new Moonie actuator, the static deformation was analysed by finite element method. Figure 2 shows the dimension of a new Moonie actuator. Figure 3 demonstrates the fourth part finite element model. Linear solid element with eight nodes was employed. Total numbers of elements and nodes are 10,557 and 12,685. Material properties of the piezoelectric element and the metal cap were set to PZT ceramic and brass, respectively. Figure 2 also shows the boundary condition. The electric potential of middle surface was set to $0.0 \mathrm{~V}$ and the electric potential of the upper and lower surfaces was set to $1.0 \mathrm{~V}$. Namely an unit positive electric voltage was applied to the upper piezoelectric plate and negative one was given to the lower.

The outer circumferential bottom was fixed along the 3rd axis direction.

Figure 4 shows the comparison of deformation under (a) positive and (b) negative electric voltages. Figure 5 shows the overall deflection curve of the new Moonie actuator. The vertical axis means the deflection of the bottom surface of the metal cap and the horizontal axis is the radial distance from a disk center. In the case of a positive electric voltage, the piezoelectric element bends on the lower side because the upper plate shrivels and the lower extends in inplane directions. By contrast the metal cap bends on the upper side. In the case of 


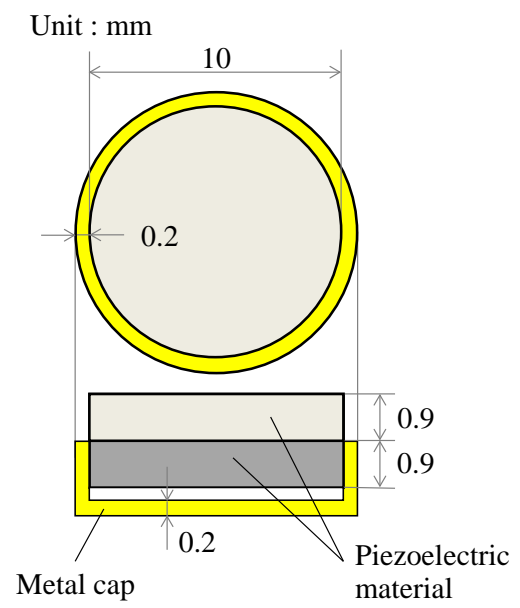

Figure 2: $\quad$ Dimension of the new Moonie actuator.

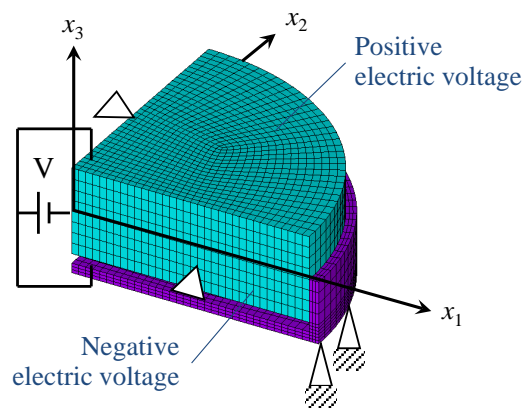

Figure 3: $\quad$ FE model and boundary condition for the new Moonie actuator.

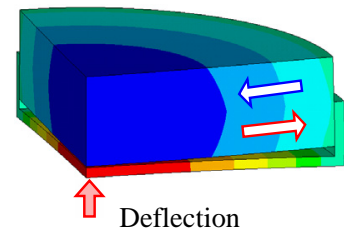

(a) $+1.0 \mathrm{~V}$

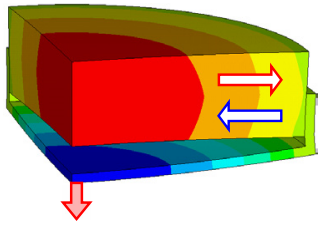

(b) $-1.0 \mathrm{~V}$

Figure 4: Deformation of the new Moonie actuator under positive and negative electric voltages. 


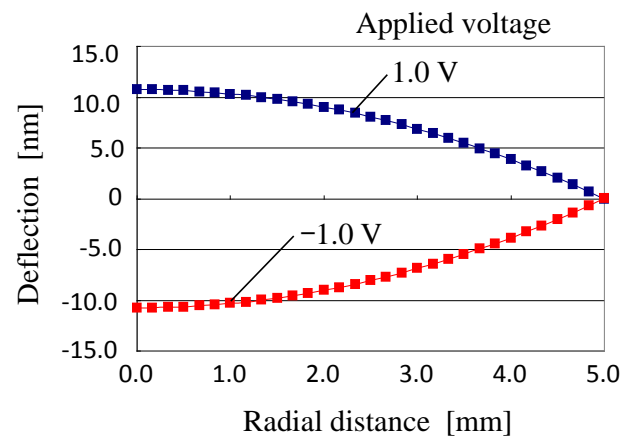

Figure 5: $\quad$ Overall deflection curve of the new Moonie actuator.

a negative, the piezoelectric element and the metal cap indicate the opposite deformation. The deflection side of metal cap was dominated by the deflection angle at the edge of piezoelectric element.

The influence of geometries of the metal cap on the overall deflection of the new Moonie actuator was investigated by changing three geometric parameters, which are adhesive thickness $(h)$, clearance $(c)$ and thickness of metal cap $\left(t_{\mathrm{m}}\right)$ as shown in Figure 6. Figure 7 shows the influence of adhesive thickness on center deflection in the case of keeping clearance and thickness of metal cap constant values, $t_{\mathrm{m}}=0.10 \mathrm{~mm}, c=0.30 \mathrm{~mm}$. Computational result indicates that the center deflection increases according to adhesive thickness. Additionally the deflection of the new Moonie actuator is considerable larger than conventional bimorph actuator without metal cap. In the same way Figure 8 shows the influence of clearance on center deflection in the case of keeping the other parameter constant, $t_{\mathrm{m}}=0.20 \mathrm{~mm}, h=0.90 \mathrm{~mm}$. The center deflection has the tendency that it becomes the largest when the clearance is almost equal to the thickness of metal cap. Figure 9 shows the influence of clearance and thickness of metal cap when both parameters are set to the same values. As their parameters become larger, the center deflection decreases. From the above computations, it is considered that the desired geometric condition is $t_{\mathrm{m}}=c=$ $0.20 \mathrm{~mm}, h=0.90 \mathrm{~mm}$ to get the largest deflection.

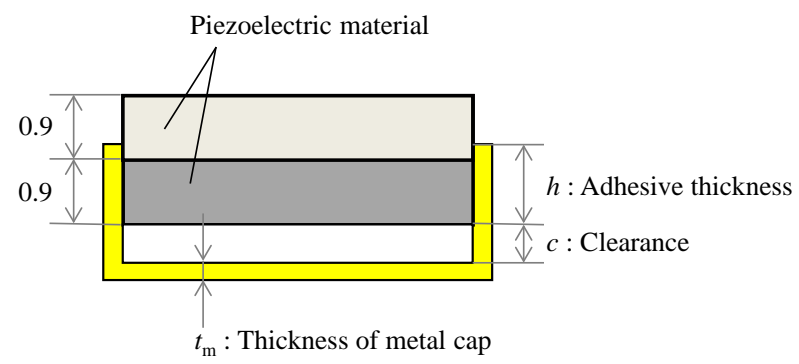

Figure 6: Geometric parameters of metal cap. 


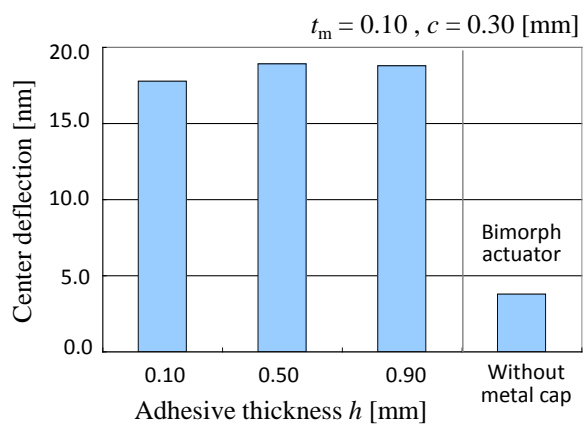

Figure 7: The influence of adhesive thickness of metal cap on center deflection.

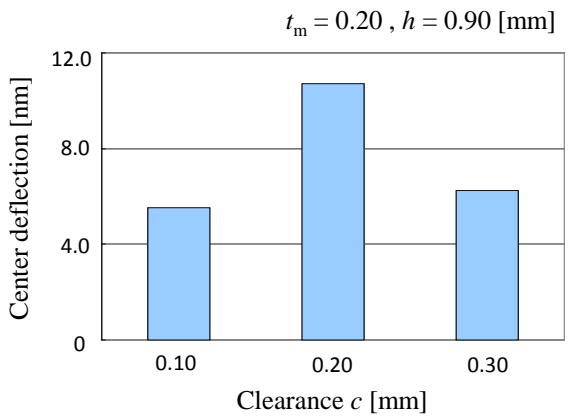

Figure 8: The influence of clearance of metal cap on center deflection.

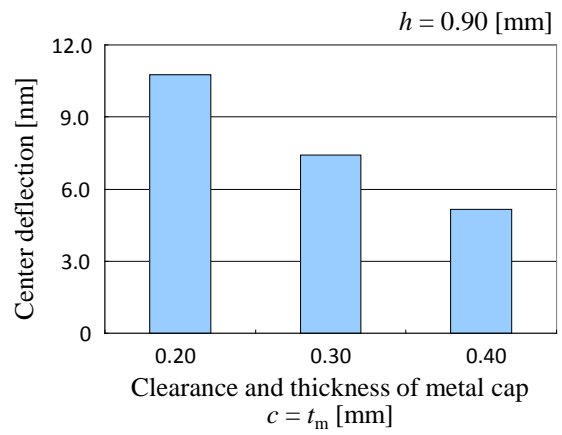

Figure 9: The influence of clearance and thickness of metal cap on center deflection. 


\subsection{Comparison of static deflection}

To verify the superiority of the proposed new Moonie actuator, the computational result of static deflection was compared with the conventional ones. Finite element modelling and the boundary condition conformed with those of the previous simulation as shown in Figure 3. Figure 10 shows the comparison of center deflection among four actuators. If compared with the monomorph actuator, the Moonie actuator has an approximate 260\% increase by addition of a metal cap. Equally the new Moonie actuator has about 290\% increase compared with the bimorph actuator. In the case of composite actuators, the increment of the new Moonie actuator attains to approximate 140\% compared with the conventional Moonie actuator. It can be confirmed that the increment of in-plane strain and the occurrence of deflection angle work efficiently for amplification of overall deflection.

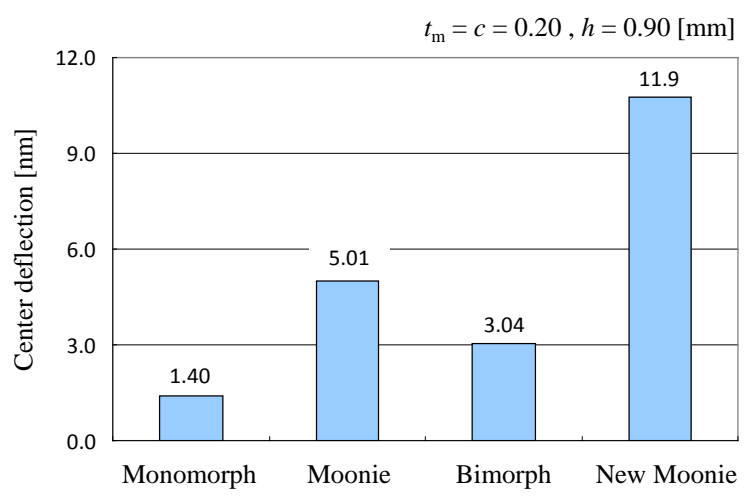

Figure 10: The comparison of center deflection among the conventional and the proposed new actuators.

\section{Performance assessment of a valve-less pump}

\subsection{Finite element analysis of Y-shaped fluid channel driven by the new Moonie actuator}

The proposed new Moonie actuator was applied to a Y-shaped fluid channel [9] and the pumping performance was evaluated through piezoelectric-fluid interaction finite element simulation. Configuration of the Y-shaped fluid channel driven by a piezoelectric actuator is shown in Figure 11. There are two reservoir tanks, which have $10 \mathrm{~mm}$ high and $0.1 \mathrm{~mm}$ diameter, at both edges of a main straight fluid channel. Another sub fluid channel joints at the center of main fluid channel with $45^{\circ}$. The main and sub fluid channels have $30 \mathrm{~mm}$ and $10 \mathrm{~mm}$ length, respectively. Both channels have $0.1 \mathrm{~mm} \times 0.1 \mathrm{~mm}$ square cross section. There is a $10 \mathrm{~mm}$ diameter fluid pool, whose top surface is bonded with a 
piezoelectric actuator, at the other edge of sub fluid channel. Fluid was assumed to be water which has $998 \mathrm{~kg} / \mathrm{m}^{3}$ density and $0.89 \mathrm{mPa} \cdot \mathrm{s}$. All of channels, reservoir tanks and fluid pool were filled with water. The water levels of right and left reservoir tanks were equal and set to $5 \mathrm{~mm}$ at the initial state. Figure 12 shows finite element model for the Y-shaped fluid channel. Total numbers of elements and nodes were 447,400 and 494,707, respectively. The cross section was divided into $10 \times 10$ elements.

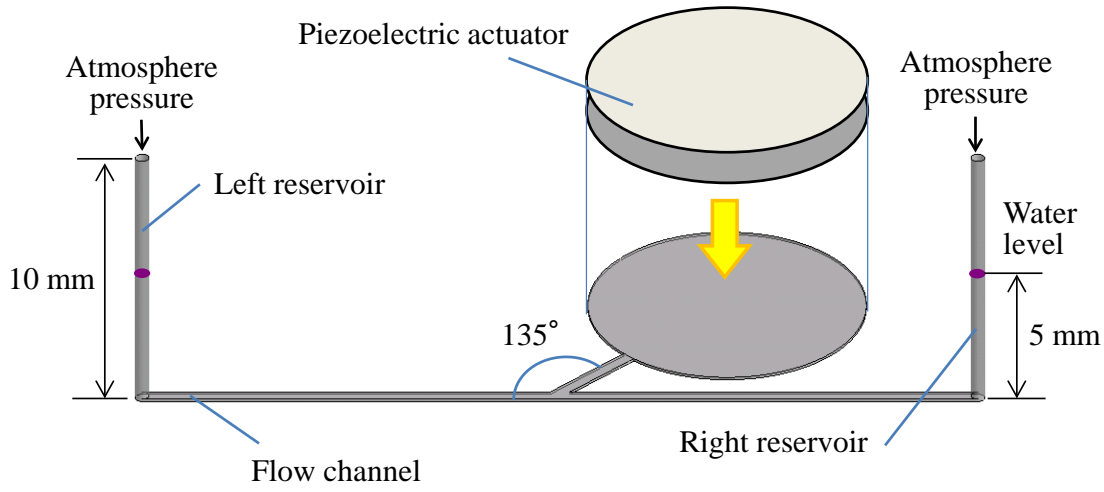

Figure 11: Y-shaped fluid channel driven by a piezoelectric actuator.

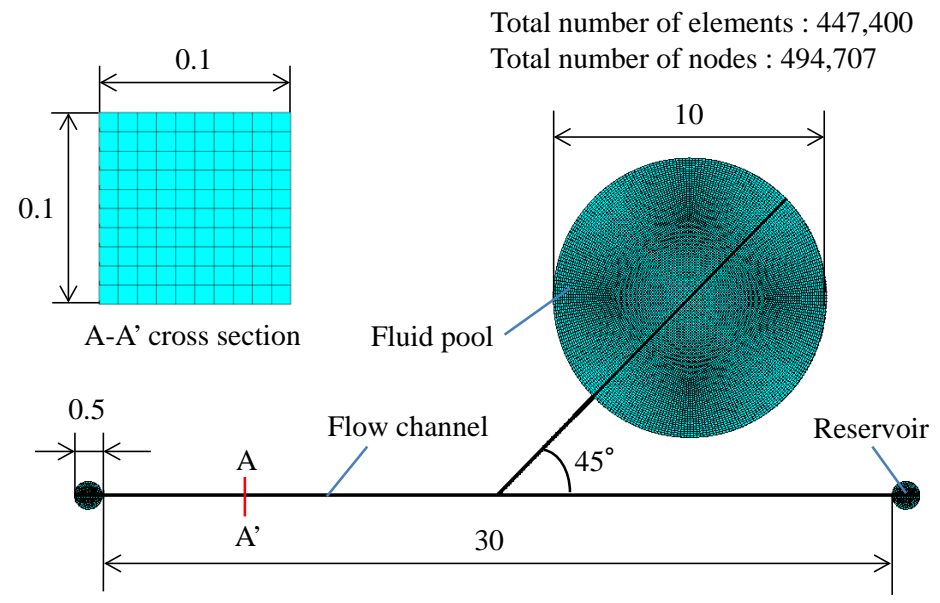

Figure 12: $\quad$ FE model for Y-shaped fluid channel.

The new Moonie actuator was bonded on the top of fluid pool and an alternating voltage was applied to it. The voltage has a peak value of $100 \mathrm{~V}$ and $50 \mathrm{~Hz}$ frequency. Figure 13 shows the variation of water levels of right and left reservoir tanks. Water levels of both tanks change periodically according to the frequency of the applied voltage. Water levels increase when the actuator bends 
on the lower side. By contract water levels decrease when the actuator bends on the upper side. If comparing water levels at the local maximum and the local minimum, the difference of water levels between two tanks becomes larger with time. The difference of water levels is an indicator of pumping performance. Figure 14 shows the time variation of water level difference between two tanks. Although there are a lot of small variations, the water level difference has the overall increasing tendency. It can be confirmed that the new Moonie actuator creates the certain pumping ability.

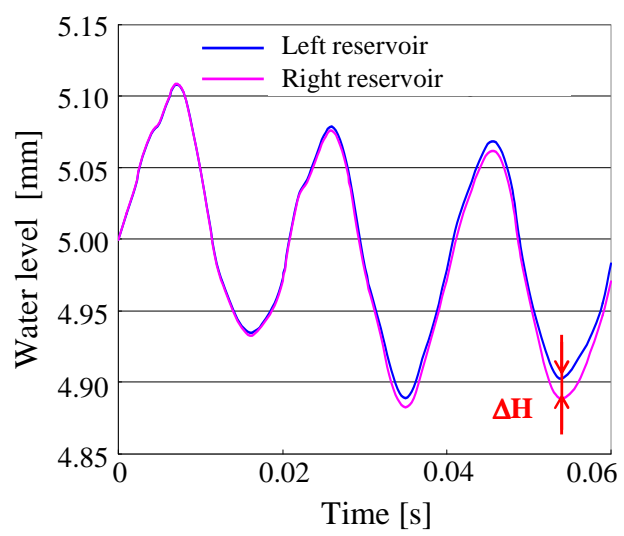

Figure 13: Time variation of water levels of right and left reservoir tanks.

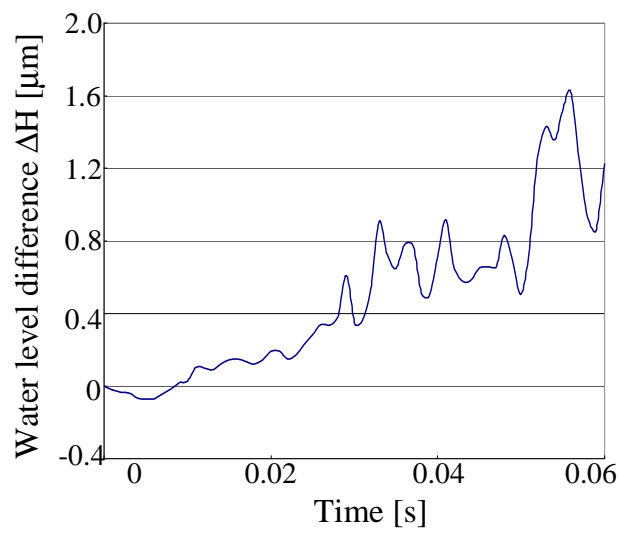

Figure 14: Time variation of water level difference between two reservoir tanks.

\subsection{Comparison of pumping performance}

To verify the superiority of the proposed new Moonie actuator, the computational result of water level difference between two reservoir tanks was compared with the conventional ones. Figure 15 shows the comparison of time 
variation of water level difference among three actuators, which are the bimorph, the Moonie and the new Moonie actuators. In the case of any actuators, there is overall increasing tendency with time. Figure 16 shows the temporal gradient of water level difference to compare the pumping ability quantitatively among three actuators. These temporal gradients were estimated from the line approximation as shown in Figure 15. Compared with the conventional Moonie actuator, the proposed new Moonie actuator accomplishes performance upgrade more than double.

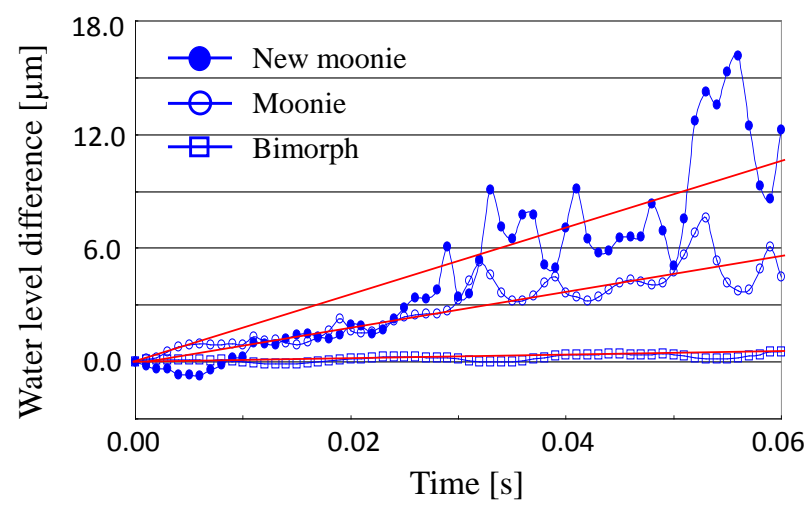

Figure 15: The comparison of time variation of water level difference among the bimorph, the Moonie and the new Moonie actuators.

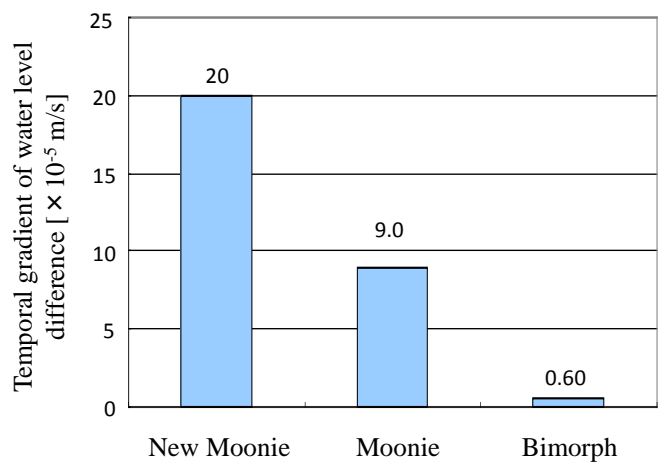

Figure 16: The comparison of temporal gradient of water level difference among the bimorph, the Moonie and the new Moonie actuators.

\section{Conclusion}

A new composite actuator called "new Moonie”, which consists of a bimorph piezoelectric actuator and a metal cap, was proposed and applied to a valve-less pump. As computational results, the proposed new Moonie actuator attains 
approximate $140 \%$ increase of the static deflection compared with the conventional Moonie actuator. Additionally the proposed actuator accomplishes upgrade of pumping ability more than double in the case of the Y-shaped fluid channel.

\section{Acknowledgements}

The authors thank master student Tomoyuki Hiramori of Osaka Institute of Technology for assistance on computations. One of the authors (Y. Uetsuji) was financially supported by a Grant-in-Aid for Young Scientists (B) (No. 22760087) from the Ministry of Education, Culture, Sports, Science and Technology of Japan.

\section{References}

[1] Xu, Q. C., Yoshikawa, S., Belsick, J. and Newnham, R. E., Piezoelectric composites with high sensitivity and capacitance for use at high pressures. IEEE Transactions on Ultrasonics, Ferroelectrics and Frequency Control, 38, pp. 634-639, 1991.

[2] Sugawara, Y., Onitsuka, K., Yoshikawa, S., Xu, Q., Newnham, R. E. and Uchino, K., Metal-ceramic composite actuators. Journal of the American Ceramic Society, 75, pp. 996 -998, 1992.

[3] Onitsuka, K., Dogan, A., Tressler, J. F., Xu, Q., Yoshikawa, S., and Newnham, R. E., Metal-ceramic composite transducer, the "Moonie". Journal of Intelligent Material Systems and Structures, 6, pp. 447-455, 1995.

[4] Meyer Jr, R. J., Dogan, A., Yoon, C., Pilgrim, S. M. and Newnham, R. E., Displacement amplification of electroactive materials using the cymbal flextensional transducer. Sensors and Actuators A: Physical, 87, pp. 157162, 2001.

[5] Nelli Silva, E. C., Nader, G., Shirahige, A. B. and Adamowski, J. C., Characterization of novel flextensional actuators designed by using topology optimization method. Journal of Intelligent Material Systems and Structures, 14, pp. 297-308, 2003.

[6] Zhang, T. and Wang, Q. M., Performance evaluation of a valve-less micropump driven by a ring-type piezoelectric actuator. IEEE Transaction on Ultrasonics, Ferroelectrics, and Frequency Control, 53, pp. 463-473, 2006.

[7] Tanaka, S. et al., Development of diffuser/nozzle based valve-less micropump, Transactions of the Japan Society of Mechanical Engineers, B 74, pp. 323-328, 2008.

[8] Kojima, Y., Okusawa, T., Tsubouchi, K., Takagi, Y. and Hamano, N., Fundamental investigation of piezo-element-driven pump feeding a trace of liquid. Transactions of the Japan Society of Mechanical Engineers, C 58, pp. 3511-3516, 1992.

[9] Kidera, M., Tsukamoto, H., Miyazaki, K., A valve-less micro pump driven by a piezoelectric device. Turbomachinery, 31, pp. 435-439, 2003. 